\title{
Pattern selection in parametrically driven arrays of nonlinear resonators
}

\author{
Eyal Kenig and Ron Lifshitz \\ Raymond and Beverly Sackler School of Physics and Astronomy, Tel Aviv University, Tel Aviv 69978, Israel \\ M. C. Cross \\ Condensed Matter Physics 114-36, California Institute of Technology, Pasadena, California 91125, USA
}

(Received 26 August 2008; published 5 February 2009)

\begin{abstract}
We study the problem of pattern selection in an array of parametrically driven nonlinear resonators with application to microelectromechanical and nanoelectromechanical systems, using an amplitude equation recently derived by Bromberg, Cross, and Lifshitz [Phys. Rev. E 73, 016214 (2006)]. We describe the transitions between standing-wave patterns of different wave numbers as the drive amplitude is varied either quasistatically, abruptly, or as a linear ramp in time. We find interesting hysteretic effects, which are confirmed by numerical integration of the original equations of motion of the interacting nonlinear resonators.
\end{abstract}

DOI: 10.1103/PhysRevE.79.026203

PACS number(s): 05.45.-a, 45.70.Qj, 62.25.- $\mathrm{g}, 85.85 .+\mathrm{j}$

\section{INTRODUCTION}

Interest in the nonlinear dynamics of microelectromechanical and nanoelectromechanical systems (MEMS and NEMS) has grown rapidly over the last few years, driven by a combination of practical needs as well as fundamental questions [1]. Lithographic fabrication technology allows the construction of large arrays of MEMS and NEMS devices (as many as 2800 to date [2]), coupled by electric, magnetic, or elastic forces. In addition, nonlinear behavior is readily observed in these devices at relatively small amplitudes of motion [3-14]. Limitations in the fabrication technology mean that individual devices will usually have slightly different resonant frequencies, and nonlinear collective effects, such as synchronization (all devices oscillating in phase) $[15,16]$ and pattern formation [17-20] (coherent response with a more complex spatial structure), have been proposed as ways of achieving useful coherent responses. Consequently, for many technological applications, there exists a practical need to understand the collective nonlinear behavior of MEMS and NEMS devices.

At the same time, the advances in the fabrication, transduction, and detection of MEMS and NEMS resonators open up an exciting new experimental window into the study of fundamental questions in collective nonlinear dynamics. Typical nonlinear MEMS and NEMS resonators are characterized by extremely high frequencies - recently going beyond $1 \mathrm{GHz}[21,22]$ —and relatively weak dissipation, with quality factors in the range of $10^{2}-10^{5}$. For such devices, transients die out rapidly, so that it is easy to attain the longtime asymptotic states, be they steady, periodic, or chaotic, and to acquire sufficient data to characterize these states well. From the theoretical point of view, the systems have the advantage that the basic physics of the individual elements is simple, and the parameters can be measured or calculated, so that the equations of motion describing the system can be established with confidence. This, and the fact that weak dissipation can be treated as a small perturbation, provide a

\footnotetext{
*Corresponding author. ronlif@tau.ac.il
}

great advantage for quantitative theoretical study. Moreover, the ability to fabricate arrays of thousands of coupled resonators opens new possibilities in the study of nonlinear dynamics of intermediate numbers of degrees of freedommuch larger than one can study in macroscopic or table-top experiments, yet much smaller than one studies when considering nonlinear aspects of phonon dynamics in a crystal.

Our current studies are motivated by the experimental work of Buks and Roukes [17], who fabricated an array of nonlinear micromechanical doubly clamped gold beams, and excited them parametrically by modulating the strength of an externally controlled electrostatic coupling between neighboring beams. The Buks and Roukes experiment was modeled by Lifshitz and Cross [19] using a set of coupled nonlinear equations of motion. They used secular perturbation theory to convert these equations of motion into a set of coupled nonlinear algebraic equations for the normal mode amplitudes of the system, enabling them to obtain exact results for small arrays, but only a qualitative understanding of the dynamics of large arrays. In order to obtain analytical results for large arrays, Bromberg, Cross, and Lifshitz [20] (henceforth BCL) studied the same system of equations, approaching it from the continuous limit of infinitely many degrees of freedom, and obtaining a description of the slow spatiotemporal dynamics of the array of resonators in terms of an amplitude equation. BCL showed that this amplitude equation could predict the initial mode that develops at the onset of parametric oscillations as the driving amplitude is gradually increased from zero, as well as a sequence of subsequent transitions to other single-mode oscillations.

The combination of many degrees of freedom and nonlinearity in the equations of motion typically leads to a large multiplicity of physically realizable solutions for fixed system parameters. This is illustrated for the particular case of two and three parametrically driven oscillators by the explicit results of Lifshitz and Cross [19]. The richness of possible solutions leads to opportunities for diverse functionality of the system, in nature or technology. On the other hand, we need to be able to predict which out of the possible solutions will be seen for a given experimental protocol, or design particular protocols such that the desired solution is the one that is formed. This is the general question of pattern selec- 
tion [23]. A common experimental protocol is to vary one or more system control parameters, usually either slowly compared with the intrinsic time scales of the dynamics, or in an abrupt step. A particular solution will usually survive (evolving adiabatically in the former case of slow parameter variation) until it becomes unstable to small perturbations, and a sequence of patterns can be predicted by analyzing these instabilities.

In this paper we investigate the sequence of single mode standing wave patterns to be expected in parametrically driven oscillator arrays, in cases where many such modes are simultaneously stable, when the strength of the driving is varied. Although the quantitative analysis could be done directly from the basic oscillator equations of motion, it is advantageous to formulate the analysis in terms of the BCL amplitude equation. This allows us to display the range of stable patterns on a reduced plot involving just two dimensionless variables (a scaled measure of the driving strength, and a scaled mode wave number), so that it is easy to deduce the general qualitative behavior on varying parameters. The specific quantitative behavior for a physical system is also easy to obtain by evaluating the corresponding scaled quantities. A change of pattern occurs when parameters vary so that the mode moves outside of the region of stable patterns on this plot, and the new pattern is predicted by analyzing the result of the instability using the BCL amplitude equation. This type of approach has been used in other pattern forming systems [24]. A unique feature of the present system is that the difference in the instabilities encountered on increasing and decreasing the (scaled) driving strength leads to the prediction of quite different sized mode jumps for the up and down sweeps.

The outline of the paper is as follows. In Sec. II we review the derivation of the amplitude equation of BCL, and in Sec. III use this equation to discuss the stability of singlemode oscillating patterns. We then study the sequence of patterns observed for a variety of time dependent sweeps of the driving strength: quasistatic variation in Sec. IV; abrupt step jumps in Sec. V; and a control parameter ramp varying linearly in time in Sec. VI. Finally, we conclude with some remarks connecting our results to those of Buks and Roukes [17] who swept the frequency rather than the driving strength.

\section{BCL AMPLITUDE EQUATION}

Lifshitz and Cross [19] modeled the array of coupled nonlinear resonators that was studied by Buks and Roukes [17] using the equations of motion

$$
\begin{aligned}
\ddot{u}_{n}+ & u_{n}+u_{n}^{3}-\frac{1}{2} \epsilon\left(\dot{u}_{n+1}-2 \dot{u}_{n}+\dot{u}_{n-1}\right) \\
& +\frac{1}{2}\left[\Delta^{2}+\epsilon h \cos \left(2 \omega_{p} t\right)\right]\left(u_{n+1}-2 u_{n}+u_{n-1}\right) \\
& -\frac{1}{2} \delta^{1 / 2}\left[\left(u_{n+1}-u_{n}\right)^{2}\left(\dot{u}_{n+1}-\dot{u}_{n}\right)-\left(u_{n}-u_{n-1}\right)^{2}\left(\dot{u}_{n}-\dot{u}_{n-1}\right)\right] \\
& =0
\end{aligned}
$$

where $u_{n}(t)$ describes the deviation of the $n$th resonator from its equilibrium, with $n=1, \ldots, N$, and fixed boundary conditions $u_{0}=u_{N+1}=0$. Detailed arguments for the choice of terms introduced into the equations of motion are discussed in Ref. [19]. The terms include an elastic restoring force with both linear and cubic contributions (whose coefficients are both scaled to 1), a dc electrostatic nearest-neighbor coupling term with a small ac component responsible for the parametric excitation at $2 \omega_{p}$ (with coefficients $\Delta^{2}$ and $\epsilon h$, respectively), and linear as well as cubic nonlinear dissipation terms. The dissipation in the system is assumed to be weak, which is used to define two small expansion parameters $\epsilon \ll 1$ and $\delta \ll 1$ by setting the linear damping rate to $\epsilon$ and the nonlinear damping coefficient to $\delta^{1 / 2}$, with a square root for later convenience. The driving amplitude is then expressed as $\epsilon h$, with $h$ of order one, in anticipation of the fact that parametric oscillations at half the driving frequency require a driving amplitude which is of the same order as the linear damping rate [25]. Both dissipation terms are taken to be of a nearest neighbor form, motivated by the experimental indication that most of the dissipation comes from the electrostatic interaction between neighboring beams.

In order to treat the system of equations (1) analytically, BCL introduced a continuous displacement field $u(x, t)$, and slow spatial and temporal scales, $X=\epsilon x$ and $T=\epsilon t$. They tried a solution in terms of a pair of counterpropagating plane waves, oscillating at half the drive frequency,

$$
\begin{aligned}
u(x, t)= & \epsilon^{1 / 2}\left\{\left[A_{+}(X, T) e^{-i q_{p} x}+A_{-}^{*}(X, T) e^{i q_{p} x}\right] e^{i \omega_{p} t}+\text { c.c. }\right\} \\
& +\epsilon^{3 / 2} u^{(1)}(x, t, X, T)+\cdots
\end{aligned}
$$

where the asterisk and c.c. denote complex conjugation, and $q_{p}$ and $\omega_{p}$ are related through the dispersion relation

$$
\omega_{p}^{2}=1-2 \Delta^{2} \sin ^{2}\left(\frac{q_{p}}{2}\right) .
$$

By substituting this ansatz (2) into the equations of motion (1) and applying a solvability condition on the terms of order $\epsilon^{3 / 2}$, BCL obtained a pair of coupled amplitude equations for the counterpropagating wave amplitudes $A_{+}$. A linear analysis of these equations shows that at the critical drive amplitude $h_{c}=2 \omega_{p}$ a particular linear combination of the two counterpropagating waves obtains a positive growth rate, forming a standing wave pattern, while the growth rate of the orthogonal linear combination remains negative. This implies that a single amplitude equation should suffice at onset, describing this standing wave pattern.

At this point it is natural to define a reduced driving amplitude $g$ with respect to the critical drive $h_{c}$ at onset by letting $\left(h-h_{c}\right) / h_{c} \equiv g \delta$, and to introduce a second ansatz,

$$
\begin{aligned}
\left(\begin{array}{l}
A_{+} \\
A_{-}
\end{array}\right)= & \delta^{1 / 4}\left(\begin{array}{l}
1 \\
i
\end{array}\right) \hat{B}(\hat{\xi}, \hat{\tau})+\delta^{3 / 4}\left(\begin{array}{l}
w^{(1)}(X, T, \hat{\xi}, \hat{\tau}) \\
v^{(1)}(X, T, \hat{\xi}, \hat{\tau})
\end{array}\right) \\
& +\delta^{5 / 4}\left(\begin{array}{c}
w^{(2)}(X, T, \hat{\xi}, \hat{\tau}) \\
v^{(2)}(X, T, \hat{\xi}, \hat{\tau})
\end{array}\right),
\end{aligned}
$$

where $\hat{\xi}=\delta^{1 / 2} X$ and $\hat{\tau}=\delta T$. Substitution of this ansatz allows 
one to obtain the correction of the solution at order $\delta^{3 / 4}$,

$$
\left(\begin{array}{c}
w^{(1)} \\
v^{(1)}
\end{array}\right)=\frac{1}{4 \omega_{p} \sin ^{2}\left(q_{p} / 2\right)}\left(\Delta^{2} \sin \left(q_{p}\right) \frac{\partial \hat{B}}{\partial \hat{\xi}}+9 i|\hat{B}|^{2} \hat{B}\right)\left(\begin{array}{c}
1 \\
-i
\end{array}\right),
$$

after which a solvability condition applied to the terms of order $\delta^{5 / 4}$, and a rescaling of all the physical quantities, yield an equation for the scaled field $B(\xi, \tau)$ of the form

$$
\frac{\partial B}{\partial \tau}=g B+\frac{\partial^{2} B}{\partial \xi^{2}}+i \frac{2}{3}\left(4|B|^{2} \frac{\partial B}{\partial \xi}+B^{2} \frac{\partial B^{*}}{\partial \xi}\right)-2|B|^{2} B-|B|^{4} B .
$$

This is the BCL amplitude equation. It is governed by a single control parameter, the reduced drive amplitude $g$, and captures the slow dynamics of the coupled resonators just above the onset of parametric oscillations. The reader is encouraged to consult Ref. [20] for a more detailed account of the derivation of the BCL equation, as well as a detailed list of all the scale factors leading to the final form of the equation.

\section{SINGLE-MODE SOLUTIONS OF THE BCL AMPLITUDE EQUATION}

The simplest nontrivial solutions of the BCL amplitude equation are steady-state single-mode extended patterns, given by

$$
B(\xi, \tau)=b_{k} e^{i(\varphi-k \xi)},
$$

with $b_{k}$ and $\varphi$ both real. This solution, when substituted back into Eqs. (5) and (4), and then into Eq. (2), yields singlemode standing-wave parametric oscillations at half the drive frequency, whose explicit form is given in the Appendix. The original boundary conditions $u(0, t)=u(N+1, t)=0$ constrain the phase $\varphi$ to be $\pi / 4$ or $5 \pi / 4$, and constrain the wave numbers of the spatial pattern to have the quantized values of $q_{m}=m \pi /(N+1)$, with $m=1, \ldots, N$.

BCL showed that the first single-mode pattern to emerge as the zero state becomes unstable is that whose wave number $q_{m}$ is closest to the wave number $q_{p}$ that is determined by the drive frequency $\omega_{p}$ through the dispersion relation (3). This determines the value of the scaled wave number in the single-mode solution (7) to be

$$
k_{0}=\left(m-q_{p} \frac{N+1}{\pi}\right) \Delta Q_{N},
$$

where $m$ is the integer closest to $q_{p}(N+1) / \pi$, and $\Delta Q_{N}$, whose explicit value is given in the Appendix, tends to zero as the size $N$ of the array of resonators tends to infinity. In this paper we are interested in secondary transitions as the initial single-mode state of wave number $k_{0}$ becomes unstable with respect to the growth of other single-mode states, whose wave numbers we label as

$$
k_{n} \equiv k_{0}+n \Delta Q_{N}
$$

In steady state, the relation between the magnitude $b_{k}$ and the wave number $k$ is found by substituting Eq. (7) into Eq.

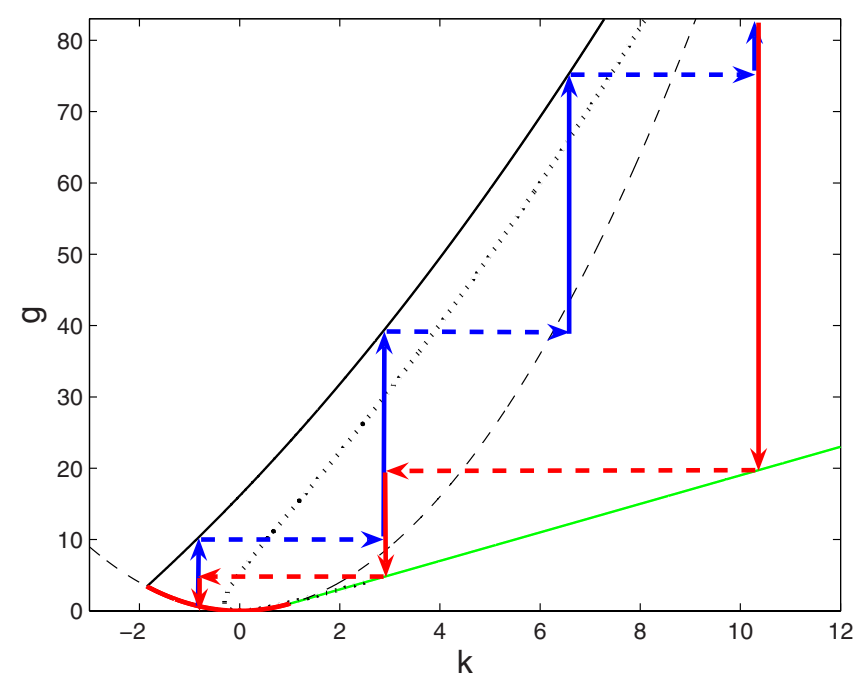

FIG. 1. (Color online) Stability boundaries of the single-mode solution (7) of the BCL amplitude equation (6) in the $g$ vs $k$ plane. Dashed line: neutral stability curve $g=k^{2}$. Dotted line: stability boundary of the single-mode solution (7) for a continuous spectrum $(Q \rightarrow 0)$. Solid lines: stability boundary of the single-mode solution for $N=92$ and the parameters $\Delta=0.5, \quad q_{p}=73 \pi / 101$, and $\epsilon=\delta=0.01$ (giving $k_{0} \simeq-0.81$ and $\Delta Q_{N} \simeq 3.70$ ). Upper boundary (solid black curve): the value of $g$ for which the eigenvalue $\lambda\left(\Delta Q_{N}\right)$ turns positive. Lower boundary for $k<1$ [solid red (dark gray) curve]: the neutral stability curve $g=k^{2}$. Lower boundary for $k>1$ [solid green (light gray) line]: the locus of saddle-node bifurcations $g=2 k-1$. Vertical and horizontal arrows mark the secondary instability transitions shown in Fig. 2 and discussed in Sec. IV; the blue upward and right-pointing arrows are for upward sweeps and the red downward and left-pointing arrow, for downward sweeps.

(6) and setting the time derivative to zero, to give

$$
b_{k}^{2}=(k-1)+\sqrt{(k-1)^{2}+\left(g-k^{2}\right)} \geqslant 0,
$$

along with a negative square-root branch which is always unstable against small perturbations [20], as can be verified by the analysis below. Linearization of the BCL amplitude equation (6) shows that the zero state with $B(\xi, \tau)=0$ - which is a solution of Eq. (6) for any value of $g$-is stable against the formation of single-mode patterns with wave number $k$ as long as $g<k^{2}$. The neutral stability curve $g=k^{2}$ is plotted as a dashed parabola in Fig. 1. Furthermore, for $k<1$ the bifurcation from the zero state to that of single-mode oscillations is supercritical, occurring on the neutral stability curve, while for $k>1$ it is subcritical, with a locus of saddle-node bifurcations located along the line $g=2 k-1$ [shown in Fig. 1 as a solid green (light gray) line], where the square root in Eq. (10) is exactly zero.

The stability of a single-mode solution (7) of wave number $k$ against an Eckhaus transition to a different single-mode solution of wave number $k \pm Q$ is found by performing a linear stability analysis of solutions of the form

$$
B(\xi, \tau)=b_{k} e^{-i k \xi}+\left[\beta_{+}(\tau) e^{-i(k+Q) \xi}+\beta_{-}^{*}(\tau) e^{-i(k-Q) \xi}\right],
$$

with $\left|\beta_{ \pm}\right| \ll 1$. When the larger of the two eigenvalues describing the growth of such a perturbation, which is given by [20] 


$$
\begin{aligned}
\lambda_{g, k}(Q)= & 2 b_{k}^{2}\left(k-1-b_{k}^{2}\right)-Q^{2} \\
& +\frac{2}{3}\left[3 Q^{2}\left(k-b_{k}^{2}\right)\left(3 k-5 b_{k}^{2}\right)+9 b_{k}^{4}\left(k-1-b_{k}^{2}\right)^{2}\right]^{1 / 2},
\end{aligned}
$$

becomes positive the single-mode solution of wave number $k$ undergoes an Eckhaus instability with respect to different single-mode solutions of wave numbers $k \pm Q$ [28].

For an infinite number of oscillators the Eckhaus instability forms the upper boundary of the stability balloon of the single-mode solutions, and also the lower boundary for $k<5 / 2$. For $k>5 / 2$ the lower boundary is the saddle node bifurcation line. For a finite number of oscillators, restricting $Q$ to be an integer multiple of $\Delta Q_{N}$ in Eq. (12) slightly shifts the Eckhaus instability lines. The upper Eckhaus boundary is shifted to larger values of $g$. The nature of the lower instability boundary now depends on the number of resonators in the array through $\Delta Q_{N}$, as well as on the wave number $k$ [20]. For $k<1$ the lower boundary will be the Eckhaus instability curve if $|k|>\Delta Q_{N} / 2$, and the neutral stability curve otherwise. From Eqs. (8) and (9) we find that the only wave number to satisfy $|k|<\Delta Q_{N} / 2$ is $k_{0}$, which means that upon decreasing $g$ the $k_{0}$ solution undergoes a continuous transition to the zero state. For $k>1$ the lower boundary will be the Eckhaus instability curve if $1<k<\left[5-3\left(\Delta Q_{N} / 2\right)^{2}\right] / 2$, and the line of saddle node bifurcations otherwise. For $\Delta Q_{N}>2$ (for the parameters used throughout this paper this corresponds to $N<172$ ) there is no portion of Eckhaus instability on the lower boundary, which is the neutral stability curve if $k<1$ and the saddle node bifurcation curve if $k>1$. These stability boundaries are shown in Fig. 1 for an infinite system and for a system of $N=92$ resonators, which is discussed next.

\section{QUASISTATIC SWEEPS OF THE CONTROL PARAMETER}

We begin by taking a close look at the switching that occurs between single-mode patterns (7) of different wave numbers $k_{n}$ as the control parameter-the reduced drive amplitude $g$-is varied quasistatically. We examine a typical situation, which is depicted within the stability balloon of single-mode solutions, shown in Fig. 1. Parameters are chosen such that the initial pattern happens to have a wave number $k_{0} \simeq-0.81$, which corresponds to the array of $N=92$ nonlinear resonators oscillating at its $m=67$ mode. Because $k_{0}<1$ we expect the pattern to grow supercritically from the zero state as the control parameter is gradually increased from $g=0$. The sequence of expected secondary transitions to single-mode patterns of wave numbers $k_{n}$ can be understood with the help of the vertical and horizontal lines drawn within the stability balloon. As $g$ reaches a value of about 10, the initial $k_{0}$ pattern undergoes an Eckhaus instability to a pattern of wave number $k_{1} \simeq 2.90$. As this occurs in the solution (7) of the amplitude equation (6) the pattern of the array of nonlinear resonators (1) switches from the 67th mode to the 68th mode via a single phase slip, in which the number of nodes in the standing-wave pattern increases ex-

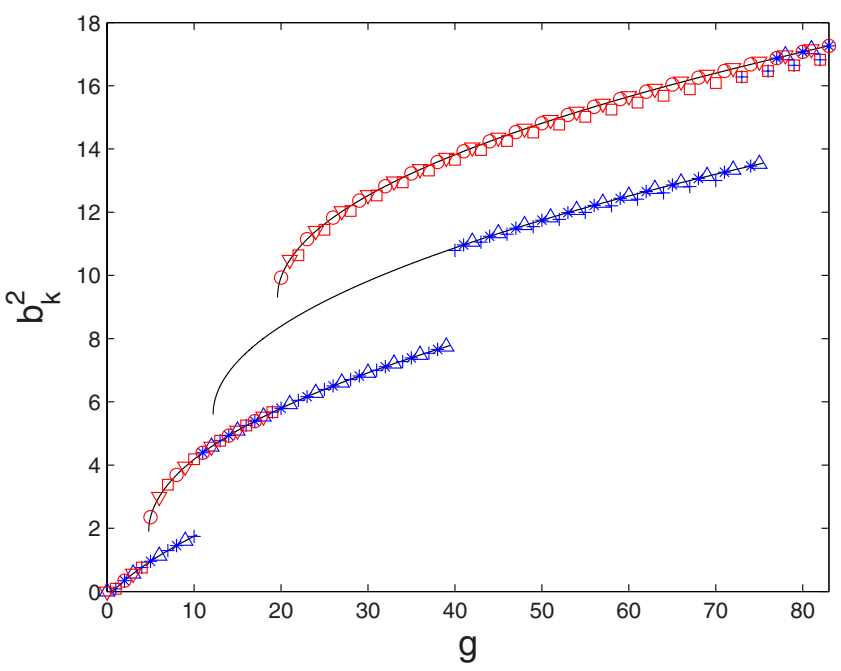

FIG. 2. (Color online) The amplitude of single-mode oscillations as a function of the reduced drive amplitude $g$. The parameters are the same as in Fig. 1. Solid lines show the analytical values (10) of the amplitudes $b_{k}^{2}$ of the modes $k_{n}$ (for $n=0, \ldots, 3$ ). Note that the $k_{0}$ mode bifurcates supercritically, whereas all the other modes start at a saddle-node bifurcations. All modes terminate at the values of $g$ for which they become Eckhaus unstable. Symbols show numerical calculations-where in the online version blue is used for upward sweeps of $g$ and red is used for downward sweeps-as follows: (a) +'s and $\square$ 's show upward and downward sweeps, respectively, of the original equations of motion (1) of the coupled resonators. (b) $\triangle$ 's and $\nabla$ 's show upward and downward sweeps, respectively, of the BCL amplitude equation (6). (c) *'s and $\bigcirc$ 's show upward and downward sweeps, respectively, of the truncated mode expansion equations (16) for the seven modes $b_{-3}$ to $b_{3}$.

actly by 1 . With the continuing increase of the control parameter $g$ the secondary pattern eventually undergoes another Eckhaus transition to $k_{2} \simeq 6.60(m=69)$, followed by a further Eckhaus transition to $k_{3} \simeq 10.30(m=70)$.

Upon decreasing the value of the control parameter $g$ back to zero, the $k_{3}$ pattern remains stable down to its saddlenode bifurcation at a value of $g$ just below 20. As we further decrease $g$, the $k_{2}$ wave number is skipped and the $k_{1}$ wave number appears, even though the control parameter is varied quasistatically. This transition is discussed in detail below. The single-mode pattern of wave number $k_{1}$ eventually reaches its saddle-node bifurcation value and is replaced by the $k_{0}$ pattern.

This sequence of secondary transitions, which is expected for such a quasistatic upward sweep of the control parameter followed by a quasistatic downward sweep, is verified numerically in Fig. 2. Solid curves show the analytical values (10) of the amplitudes $b_{k}^{2}$ of the modes $k_{n}(n=0 \ldots 3)$, plotted in the region in which the corresponding single-mode solutions (7) are stable. Superimposed symbols show the numerical solution of both the original equations of motion (1) for $N=92$ resonators, and the BCL amplitude equation (6), for a quasistatic sweep of the control parameter from $g=0$ up to $g=85$ and back down to $g=0$. We note that in order to satisfy the boundary conditions when integrating the BCL amplitude equation, both the $\delta^{1 / 4}$ and $\delta^{3 / 4}$ terms of Eq. (4), when substituted into the expression $A_{+} e^{-i q_{p} x}+A_{-}^{*} e^{i q_{p} x}$ in Eq. (2), must 
be set to zero separately at the boundaries. This yields a pair of conditions on $B$ and its derivative, at the boundaries, of the form

$$
\begin{gathered}
B e^{-i q_{p} x}-i B^{*} e^{i q_{p} x}=0, \\
\frac{\partial B}{\partial \xi} e^{-i q_{p} x}+i \frac{\partial B^{*}}{\partial \xi} e^{i q_{p} x}=0 .
\end{gathered}
$$

To study the actual process of an Eckhaus transition as it takes place, we expand the general solution of the BCL amplitude equation in the linear modes of the array

$$
B(\xi, \tau)=\sum_{n} b_{n}(\tau) e^{i\left(\pi / 4-k_{n} \xi\right)}
$$

where $k_{n}$ is defined in Eq. (9), as was done, for example, in a similar situation by Kramer et al. [24]. To satisfy the boundary conditions, as mentioned above for the single-mode solution (7), we take each mode amplitude to be zero at the boundaries by setting all the phases in Eq. (15) to $\pi / 4$, thus taking the amplitudes $b_{n}$ to be real, keeping in mind that they can be either positive or negative. Substituting a truncated mode expansion (15) containing a finite number of modes around $k_{0}$ into the BCL amplitude equation (6) allows us to replace this partial differential equation with a finite number of ordinary differential equations for the coupled mode amplitudes,

$$
\begin{aligned}
\frac{d b_{n}}{d \tau}= & \left(g-k_{n}^{2}\right) b_{n}+2 \sum_{m, p}\left(k_{p}-1-\frac{m-n}{3} \Delta Q_{N}\right) b_{m} b_{p} b_{m+p-n} \\
& -\sum_{m, l, p, r} b_{m} b_{l} b_{p} b_{r} b_{m-l+p+r-n} .
\end{aligned}
$$

Note that if all mode amplitudes except $b_{0}$ are set to zero we obtain a single equation with $n=m=p=l=r=0$, whose steady-state solution is the same as the single-mode solution of BCL (10).

As shown in Fig. 2, we can capture the sequence of Eckhaus transitions from the $k_{0}$ pattern up to the $k_{3}$ pattern, and back down to the $k_{0}$ pattern through the saddle nodes, by integrating seven coupled ordinary differential equations (16) (calculated symbolically using MATLAB) that correspond to a truncated mode expansion [15] containing the seven modes from $n=-3$ to $n=3$. The results agree very well with those obtained by integrating the full BCL amplitude equation as well as the original equations of motions for the resonators, yet the solution in terms of a truncated mode expansion allows us to inspect the transitions between patterns in greater detail.

We take a closer look at the transient behavior during the first Eckhaus transition from the initial $k_{0}$ pattern to the $k_{1}$ pattern by plotting the time evolution of the four largest modes during this Eckhaus transition, as shown in Fig. 3. One can observe the decay of the unstable mode amplitude $b_{0}$ followed by the growth of $b_{1}$ to its steady-state value. One can also see that during the transient the amplitude of the unstable mode $b_{-1}$ becomes nonzero. Its participation in the Eckhaus transition from the $k_{0}$ pattern to the $k_{1}$ pattern is essential, as can be verified by considering these two modes

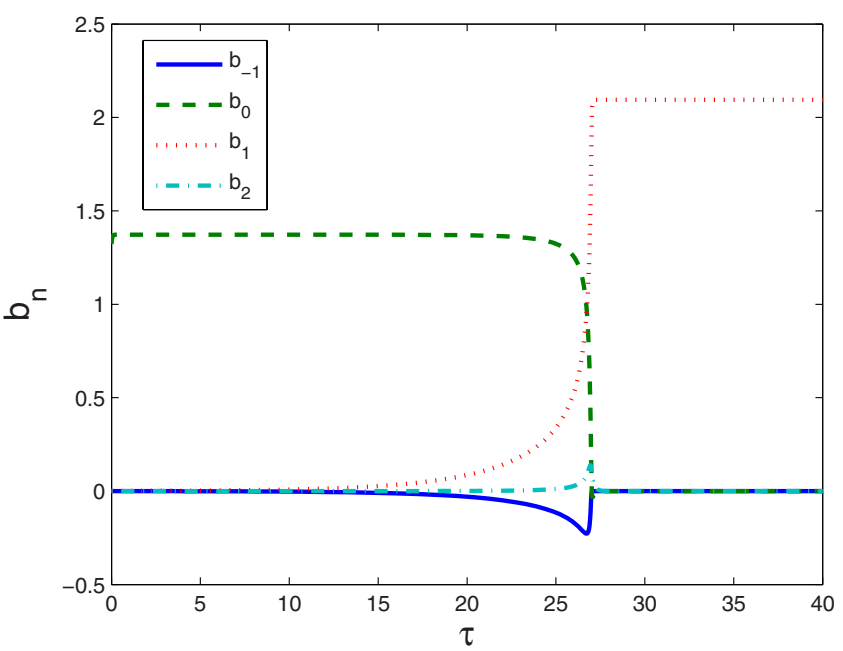

FIG. 3. (Color online) Time evolution of the amplitudes of the four largest modes that participate in the Eckhaus transition from the initial $k_{0}$ pattern to the $k_{1}$ pattern, obtained by a numerical integration of the seven truncated mode equations (16), for modes $b_{-3}-b_{3}$, using the same parameters as in Fig. 1 . The value of the control parameter is changed from $g=10$ to $g=11$ at $\tau=0$, causing the initial $k_{0}$ pattern to become unstable. The decay of the amplitude $b_{0}$ is followed by the rise of $b_{1}$ to its expected steady-state value (10), where it is clearly seen that during the transition other modes-including the unstable $k_{-1}$ mode-have a nonzero amplitude.

alone in a truncated expansion. Limiting the expansion to $b_{0}$ and $b_{1}$ suppresses the Eckhaus transition, and the $k_{0}$ pattern remains stable as $g$ exceeds its expected value for the Eckhaus instability. The Eckhaus transition is observed only when the $k_{-1}$ mode is included as well, corresponding to the stability calculation, performed earlier for the state given by Eq. (11).

One might naively expect that the same mechanism causes the transition from the $k_{3}$ pattern to the $k_{1}$ pattern at $g=19$ through a double phase slip, however, this is not the case. Figure 4 reveals the transient processes on a downward sweep of $g$ just below the saddle node at $g=19$. As $g$ crosses the saddle node bifurcation point, the amplitude $b_{3}$ drops abruptly to zero. As can be seen from Eq. (16), in the zero displacement state the linear growth rates of the solutions (15) are $g-k_{n}^{2}$, so the $k_{0}$ pattern has the largest possible growth rate and it outgrows the other modes until its amplitude approaches the steady state value (10). However, according to the eigenvalue (12), at this value of $g$ the $k_{0}$ pattern is Eckhaus unstable with respect to the $k_{1}$ pattern-notice the characteristic evolution of the modes around $\tau=3$ in Fig. 4 corresponding to the Eckhaus instability (cf. around $\tau=25$ in Fig. 3). Thus the $k_{1}$ mode is ultimately the selected pattern.

\section{ABRUPT CHANGE OF THE CONTROL PARAMETER}

For a quasistatic increase of $g$ the Eckhaus instability leads to a single phase slip event and a jump by 1 of the mode number. This is no longer always the case for more 


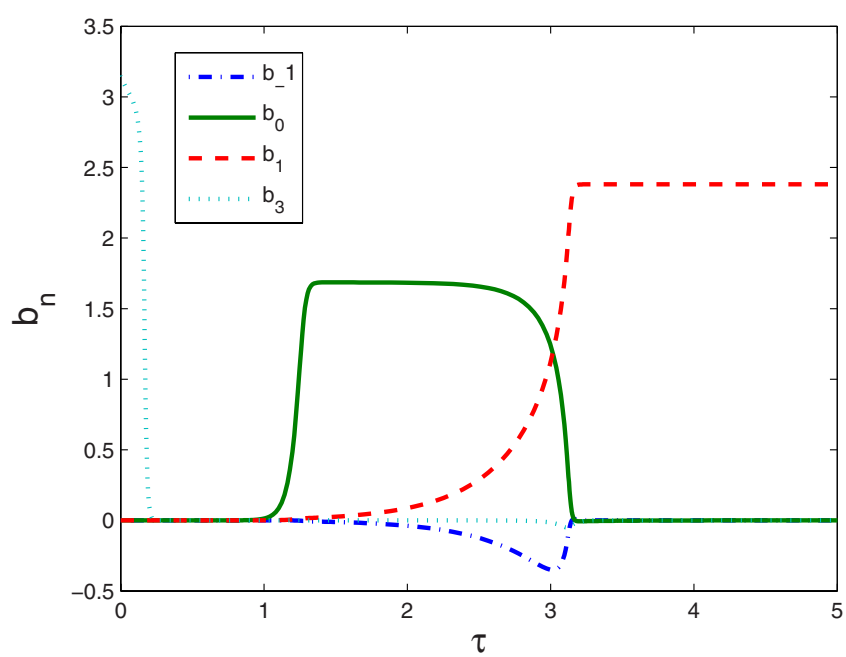

FIG. 4. (Color online) Time evolution of the amplitudes of the four largest modes as the control parameter is changed from $g=20$ to $g=19$, obtained by a numerical integration of the seven truncated mode equations (16), for the amplitudes $b_{-3}-b_{3}$, using the same parameters as in Fig. 1. As the value of $g$ drops below the saddle node value of the $k_{3}$ wave number, its amplitude drops abruptly to zero. Then, the smallest possible wave number which has the largest linear growth rate over the zero solution, $k_{0}$, grows to reach its steady-state value (10). Nevertheless, after a short transient the $k_{0}$ pattern decays through an Eckhaus instability and the $k_{1}$ pattern grows to its steady-state value.

rapid variations of $g$ [29]. In this section we consider the question of pattern selection after an abrupt jump in $g$, so that single-mode states of different wave numbers compete with each other after the system is initiated in an Eckhaus unstable state. We consider a scenario in which the system is initiated in the $k_{0}$ single-mode state (7), after which the control parameter $g$ is abruptly increased so that the $k_{0}$ wave number is no longer stable, while many other wave numbers become simultaneously stable. In order to predict the singlemode pattern that is selected we use our previous expression (12) for the eigenvalue $\lambda_{g, k_{0}}(Q)$ to calculate the linear growth rate of perturbations of single-mode patterns of wave number $k_{0}+Q$. In Fig. 5 we plot $\lambda_{g, k_{0}}(Q)$ as a function of $Q$ for four different values of $g$, illustrating the dependence of the fastest growing wave number $k_{0}+Q_{\max }$ on $g$. The wave number $k_{0}+Q_{\max }$ with the largest linear growth rate $\lambda_{\max }$, which is expected to overcome all other modes, is obtained by finding the maximum of $\lambda_{g, k_{0}}(Q)$ as a function of $Q$, yielding

$$
\begin{aligned}
Q_{\text {max }}^{2}= & \left(3 k_{0}^{2}-5 b_{k_{0}}^{2} k_{0}-3 b_{k_{0}}^{2}+2 b_{k_{0}}^{4}\right) \\
& \times \frac{\left(3 k_{0}^{2}-11 b_{k_{0}}^{2} k_{0}+3 b_{k_{0}}^{2}+8 b_{k_{0}}^{4}\right)}{3\left(k_{0}-b_{k_{0}}^{2}\right)\left(3 k_{0}-5 b_{k_{0}}^{2}\right)},
\end{aligned}
$$

and

$$
\lambda_{\max }=\frac{\left(3 k_{0}^{2}-5 b_{k_{0}}^{2} k_{0}-3 b_{k_{0}}^{2}+2 b_{k_{0}}^{4}\right)^{2}}{3\left(k_{0}-b_{k_{0}}^{2}\right)\left(3 k_{0}-5 b_{k_{0}}^{2}\right)},
$$

where $b_{k_{0}}$ is the steady-state amplitude of the unstable $k_{0}$ mode, as given by Eq. (10), that depends on the actual value of $g$.

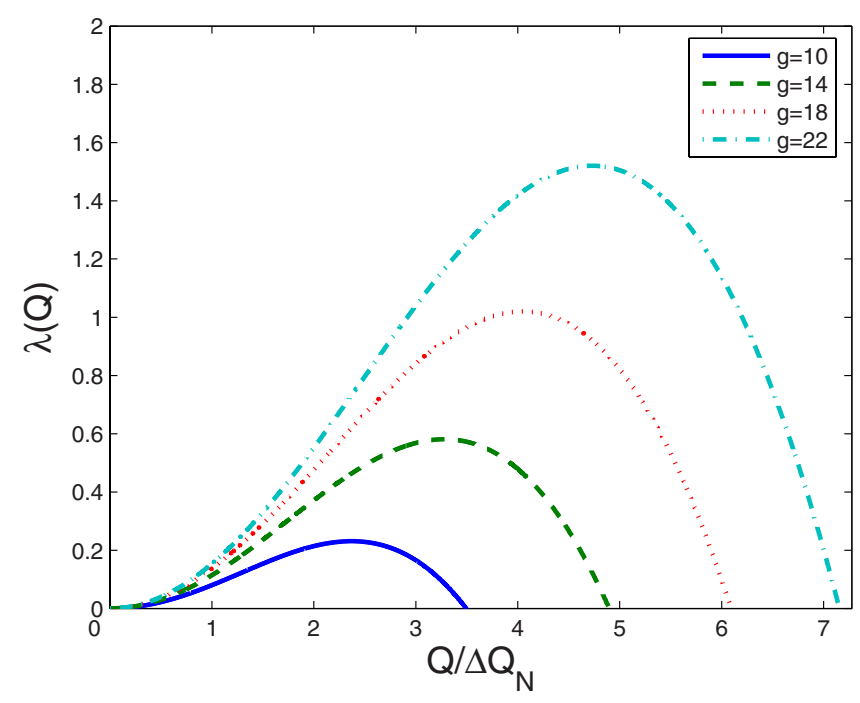

FIG. 5. (Color online) The linear growth rate $\lambda_{g, k_{0}}(Q)$ as a function of the wave number shift $Q$, plotted for different values of the control parameter $g$. The horizontal axis labels $Q$ in units of $\Delta Q_{N}$, which for a finite system of $N=500$ resonators, with $k_{0} \simeq-0.075$, has the value of $\Delta Q_{N} \simeq 0.69$ (all other parameters are the same as in Fig. 1). The Eckhaus instability of the initial $k_{0}$ pattern occurs for these parameters at $g \simeq 5$.13.

For a finite system the selected wave number is expected to be the $k_{n}$-defined in Eq. (9) — which has the largest linear growth rate. The Eckhaus instability is triggered by random small-amplitude noise. In our finite system the difference between growth rates of different modes is expected to be sufficiently large so that by the time nonlinear effects are important, the amplitude of the fastest growing mode far exceeds those of other destabilizing Eckhaus modes, and it will reach its steady state value. Figure 6 shows $Q_{\max }$ for an infinite system and for a finite system of $N=500$ resonators, where the two curves should tend to one another as $N \rightarrow \infty$. These predictions for the selected wave numbers are verified numerically by integrating the BCL amplitude equation (6), as well as the original equations of motion (1) of the coupled resonators. We note that for the parameters used the stability balloon contains about ten modes for each of the values taken for the control parameter. For $g=21$, for example, all modes with wave numbers from $k_{3}$ to $k_{16}$ are stable. Finally, by following the amplitude of the growing mode as a function of time in the numerical solution of the BCL amplitude equation (6), it is possible to extract the linear growth rate of the mode numerically. We have done so and found that the numerically calculated growth rates agree to within $2 \%$ with the analytical values of $\lambda_{g, k_{0}}\left(Q_{\max }\right)$.

\section{RAMPS OF THE CONTROL PARAMETER}

We finish by considering a scenario in which the control parameter $g$ varies smoothly with time-this is often called a control parameter ramp. To simplify the analysis we consider slow variation in time, where $d g / d \tau \ll 1$, so that we can use the expressions (7) and (10), obtained earlier for the steadystate single-mode solutions of the BCL amplitude equation, 


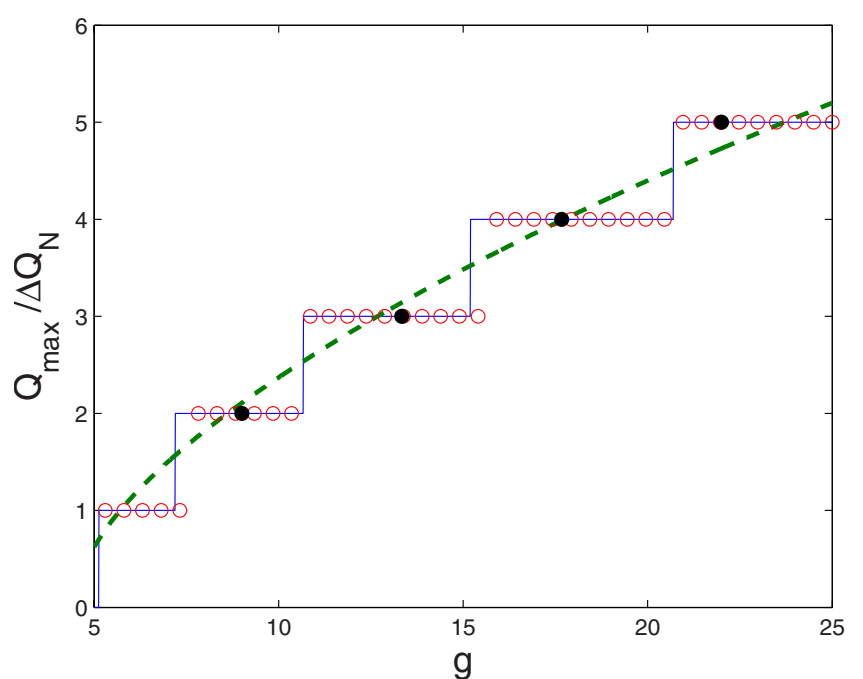

FIG. 6. (Color online) The wave number shift $Q_{\max }$ with the maximal growth rate (in units of $\Delta Q_{N}$ ) as a function of $g$. The blue solid line shows $Q_{\max }$ for the same parameters used in Fig. 5, and the green dashed line gives $Q_{\max }$ for an infinite system (17). The open red circles are the wave number shifts that are observed numerically by integrating the BCL amplitude equation (6). The full black circles are wave number shifts that are obtained by a numerical integration of the original equations of motion (1), calculated for select values of $g$. The numerical calculations are initialized with the $k_{0}$ solution and random small-amplitude noise, to initiate the growth of competing patterns. We emphasize that the results of the numerical solution of the BCL amplitude equation (6) are not sensitive to the noise amplitude as long as it is sufficiently small.

with a simple replacement of the previously constant $g$ by a time dependent $g(\tau)$,

$$
\begin{gathered}
B(\xi, \tau)=a_{n}(\tau) e^{i\left(\varphi-k_{n} \xi\right)} \\
a_{n}(\tau)^{2}=\left(k_{n}-1\right)+\sqrt{\left(k_{n}-1\right)^{2}+\left(g(\tau)-k_{n}^{2}\right)} .
\end{gathered}
$$

Thus $a_{n}(\tau)$ would be the steady-state amplitude of the pattern with wave number $k_{n}$ if the drive were varied quasistatically to its instantaneous value at time $\tau$. For ramps that are not quasistatic we expect the actual amplitude, which we denote as $\bar{a}_{n}(\tau)$, to lag behind its expected value for a quasistatic ramp. This time lag phenomenon is known from experiments measuring the heat flow in a Rayleigh-Bénard cell [26].

The specific scenario we examine is one in which the control parameter increases linearly in time from zero, $g=\alpha \tau$ with $\alpha \ll 1$. Initially, the system is expected to evolve to the single-mode state (19) with wave number $k_{0}$. As $g$ increases, this solution becomes Eckhaus unstable and a transition is expected to a different pattern, which eventually becomes Eckhaus unstable as well. It is the first of these Eckhaus transitions that we treat analytically below, as well as test numerically using the BCL amplitude equation. In order to obtain interesting mode competition, even for $\alpha \ll 1$, we increase the number of resonators to $N=1230$, thus increasing the number of stable single-mode solutions for any particular value of $g$. For such a number of resonators we no longer perform numerical calculations on the original

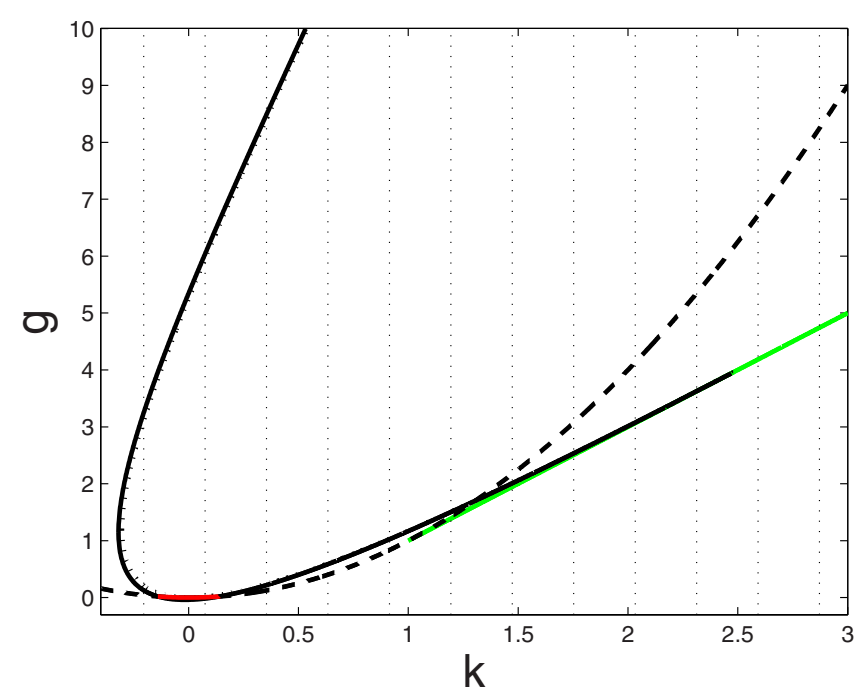

FIG. 7. (Color online) Stability balloon for an array of $N=1230$ resonators and the same parameters as in Fig. 1, which yields $k_{0} \simeq 0.075$ and $\Delta Q_{N} \simeq 0.28$. The values of $k_{n}$ for $n=-1, \ldots, 10$ are marked with vertical dotted lines. The thick dashed line is the neutral stability curve $g=k^{2}$, and the segment in which it is the lower boundary $|k|<\Delta Q_{N} / 2$ is marked by a solid red (dark gray) line. The black dotted and solid curves (which are almost indistinguishable) are the Eckhaus boundaries for an infinite and a finite system, respectively. The solid green (light gray) line is the saddle node $g=2 k-1$ which is the lower boundary of oscillations for $k>2.47$.

coupled equations of motion (1). The stability balloon for $N=1230$ resonators is shown in Fig. 7. Due to the large number of resonators, the Eckhaus boundaries for infinite and finite systems are almost the same. The dashed vertical lines mark the values of possible wave numbers $k_{n}$ for $n=-1 \ldots 10$. For $n=0(k \simeq 0.075)$ the lower boundary of oscillations is the neutral stability curve, for $n=1, \ldots, 8$ the lower boundary is the Eckhaus instability curve, and for $n \geqslant 9(k \simeq 2.5)$ it is the saddle node line.

A typical response of the system to a linear ramp of the drive amplitude is shown in Fig. 8 for a ramp rate of $\alpha=10^{-4}$. One clearly sees the amplitude of the $k_{0}$ mode, which forms initially from the zero state, becoming Eckhaus unstable around $g \simeq 7$ and undergoing a double phase slip to the $k_{2}$ mode. Thin black lines show the quasistatic values (20) of the amplitudes of these two modes as a function of $g(\tau)$, while the blue dot-dashed curve and the green dashed curve show the actual values of these two amplitudes as obtained by Fourier transforming the numerical solution of the BCL equation. The curves are distinguishable from each other only at very early times, shown in the inset of Fig. 8, clearly demonstrating the time it takes the actual amplitude $\bar{a}_{0}(\tau)$ to "catch up" with the quasistatic value $a_{0}(\tau)$, from the zero displacement state. After this initial time lag the system responds sufficiently quickly so that the ramp becomes effectively quasistatic. The only points where the time dependence of the ramp is still evident are the Eckhaus instability points at which different ramp rates are expected to lead to different transitions. A typical sequence of such transitions is shown in Fig. 9 for a ramp rate of $\alpha=10^{-5}$. 


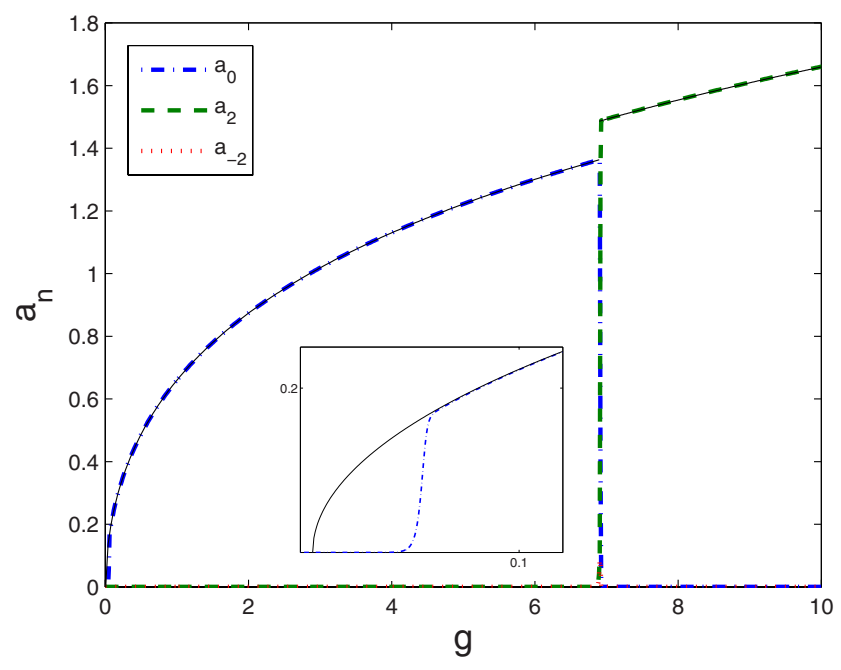

FIG. 8. (Color online) The three relevant Fourier amplitudes of the numerical solution of the amplitude equation (6) for $g=10^{-4} \tau$ and the same parameters as in Fig. 7. The quasistatic values of the amplitudes (20) are plotted in thin black lines. For $\alpha=10^{-4}$ we expect a double phase slip from the $k_{0}$ pattern to the $k_{2}$ pattern as can be inferred from Fig. 11. The inset demonstrates the time lag at early times between the actual amplitude of the $k_{0}$ mode and its quasistatic value.

To analytically predict the first Eckhaus transition, given the ramp rate $\alpha$, it is useful to introduce a more compact notation for the eigenvalues (12), which now depend on time [30], denoting $\lambda_{g(\tau), k_{0}}\left(n \Delta Q_{N}\right) \equiv \lambda_{n}[g(\tau)]$. The first five of these eigenvalues are plotted in Fig. 10 as a function of $g(\tau)$. Note that as $n$ increases, the corresponding eigenvalue $\lambda_{n}$ becomes positive at a later point in time, which we denote as $\tau_{n}$, but grows more rapidly than the smaller- $n$ eigenvalues. At time $\tau_{n}$ the amplitude of the $k_{n}$ mode is expected to start

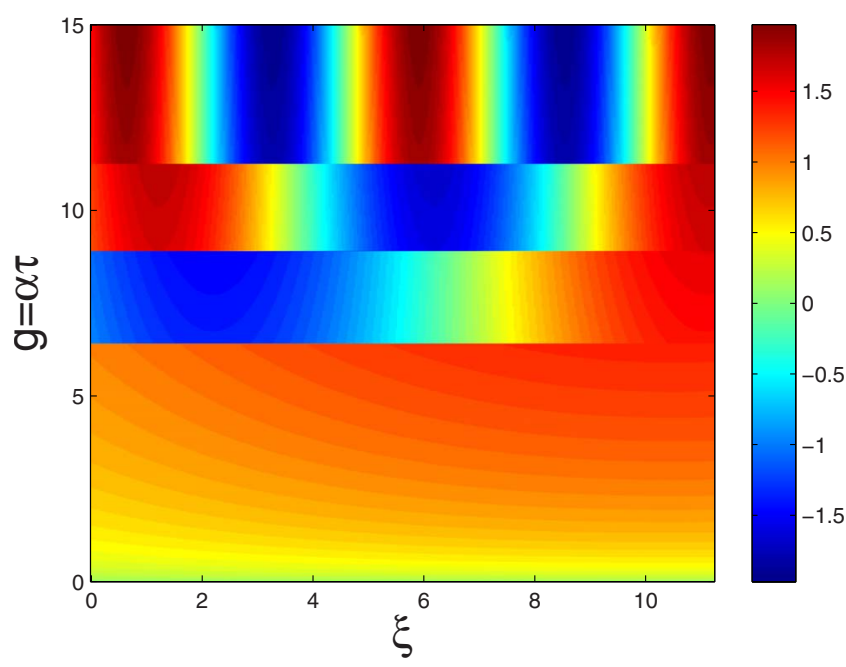

FIG. 9. (Color online) The real part of $B(\xi, \tau)$, obtained by a numerical integration of the BCL amplitude equation (6) for $g=10^{-5} \tau$, using the same parameters as in Fig. 7. The initial zero state $B(\xi, 0)=0$ evolves into the $k_{0}$ state, which then undergoes a sequence of Eckhaus transitions as $g$ increases in time-the first two transitions involve single phase slips, while the third involves a double phase slip.

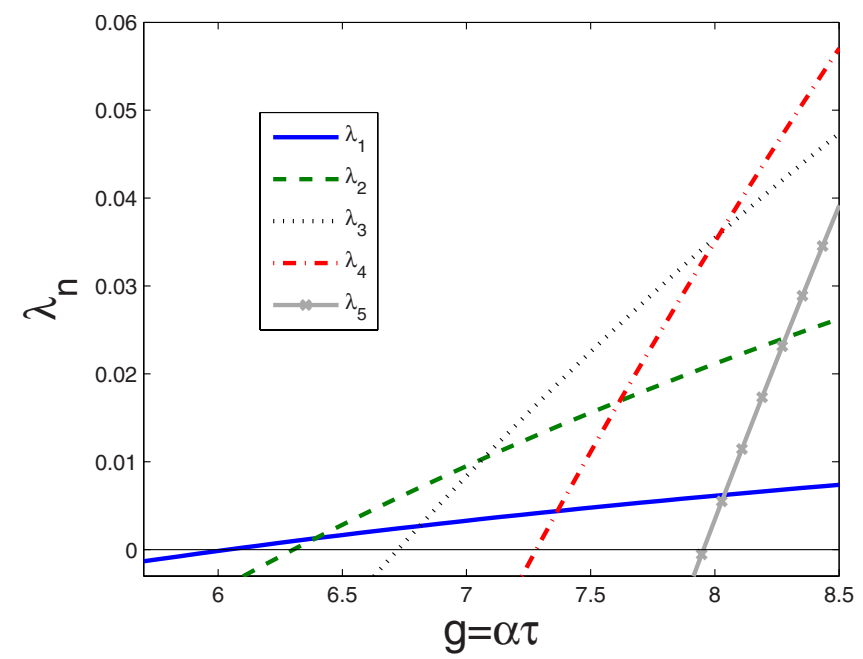

FIG. 10. (Color online) The first five eigenvalues $\lambda_{n}$, plotted as a function of $g=\alpha \tau$ using the parameters of Fig. 7. As $\lambda_{1}$ turns positive the $k_{0}$ solution becomes Eckhaus unstable, but the selected pattern depends on the ramp rate $\alpha$ as explained in the text.

growing from its initial value $\bar{a}_{n}\left(\tau_{n}\right)$, which in a real physical system is set by the noise floor. In our analysis below we take this initial value to be the same as the accuracy of the numerical routine that is used for integrating the BCL equation. Once the $k_{n}$ pattern starts growing it competes with all the other single-mode patterns with positive growth rates. We expect the pattern that is eventually selected to be the one whose amplitude is first to reach the quasistatic value $a_{n}(\tau)$, given by Eq. (20). Thus lower- $n$ modes have an advantage for small ramp rates $\alpha$ because their growth rates become positive earlier. At higher ramp rates, due to the time-lag phenomenon shown above, the higher- $n$ modes have an advantage because their eigenvalues increase more rapidly in time. This gives rise to an interesting competition between the possible stable patterns. A similar situation was observed in a system described by the stochastic time-dependent Ginzburg-Landau equation [27].

Owing to the slow ramp rates, and the fact that the second eigenvalue associated with each mode remains negative, we can estimate the growth of the $n$th amplitude, in the linear regime, from its initial value at $\tau_{n}$ to be

$$
\bar{a}_{n}(\tau)=\bar{a}_{n}\left(\tau_{n}\right) e^{\sigma_{n}\left(\tau, \tau_{n}\right)},
$$

where

$$
\sigma_{n}\left(\tau, \tau_{n}\right)=\int_{\tau_{n}}^{\tau} \lambda_{n}\left[g\left(\tau^{\prime}\right)\right] d \tau^{\prime} .
$$

A comparison of these expressions for $\bar{a}_{n}(\tau)$ for the different patterns allows us to determine which is the first to reach its quasistatic value $a_{n}(\tau)$, and provides a simple scheme for predicting the selected pattern following the Eckhaus instability of the initial $k_{0}$ pattern. These analytical predictions for the selected pattern $k_{n}$ are shown as a function of the ramp rate $\alpha$ in Fig. 11, and are nicely verified by numerical integration of the BCL amplitude equation (6). As expected, for small values of $\alpha$ there is a single phase slip to the pattern 


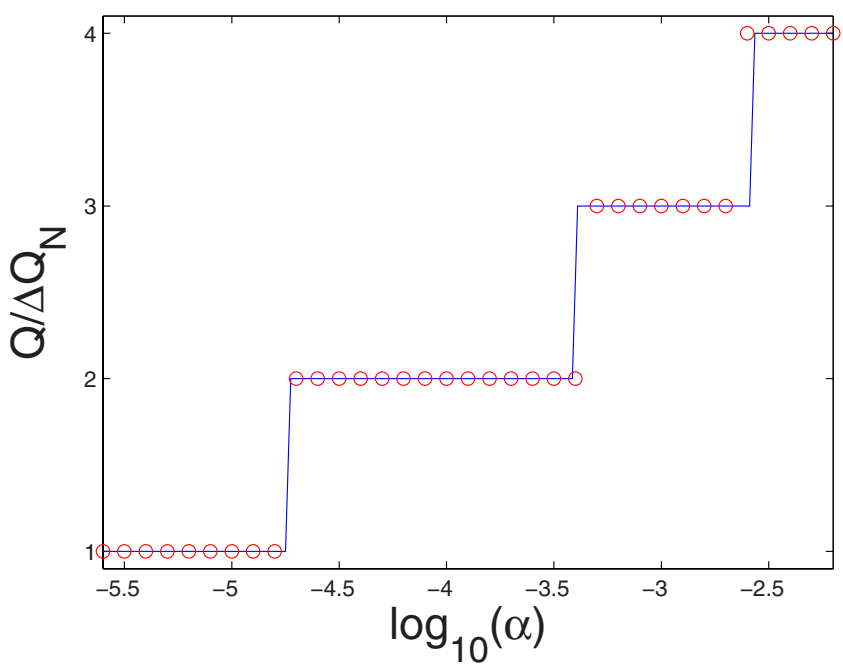

FIG. 11. (Color online) The number of phase slips $Q / \Delta Q_{N}$, that are observed following the Eckhaus instability of the initial $k_{0}$ pattern, plotted as a function of the ramp rate $\alpha$ for a linear ramp of the drive $g=\alpha \tau$. Parameters are the same as in Fig. 7. Red circles are the actual values observed in the numerical integration of the BCL amplitude equation (6). The blue line shows the predicted values from the linear analysis described in the text, where the initial amplitude of each mode, when its eigenvalue becomes positive, is taken to be $\bar{a}_{n}\left(\tau_{n}\right)=10^{-12}$, which is the accuracy of the time integration in the numerical solution of Eq. (6).

with wave number $k_{1}$. As $\alpha$ is further increased this changes to a double phase slip to the $k_{2}$ pattern (as demonstrated earlier in Fig. 8), followed by a transition to the $k_{3}$ pattern, and so on.

\section{CONCLUSIONS}

We have investigated the sequence of single-mode standing-wave patterns to be expected in one-dimensional arrays of parametrically driven oscillators for time varying drive strengths. An amplitude equation approach provides a general treatment in terms of a universal stability diagram on a plot with scaled versions of the driving strength and wave number as axes. This immediately shows the type of instability that will be encountered on varying parameters, and gives qualitative insights on the mode jumps to be expected. For example, for quasistatic parameter variations, we find that the jump in the mode number is always unity if the control parameter is increased so that the Eckhaus instability operates, but larger jumps are often seen if the control parameter is decreased so that a saddle node bifurcation occurs. For more rapid increases in the control parameter larger jumps in the mode number may also occur, and can be predicted simply from the eigenvalue equation for the Eckhaus instability. We give explicit results for examples of an abrupt jump and a slow temporal ramp in the control parameter. In all cases we checked, simulations of the original oscillator equations of motion confirm the results based on the amplitude equation.

In the Buks and Roukes experiments on parametrically driven oscillator arrays [17] which motivated this study the frequency of the drive was swept, rather than the strength of the driving. Since the drive frequency is involved in setting the wave number of the resonant mode that goes unstable, and these two parameters are involved in a complicated way in the expressions for the scaled drive and wave number variables (see BCL), it is more difficult to display the variation on the scaled stability plot of Fig. 1. For quasistatic or abrupt variations this is immaterial, since the behavior is determined by where the stability boundary is crossed in the former case, or the relationship of the final point on the stability plot to the stability boundaries in the latter case. These can be estimated quite easily, so that the behavior for quasistatic or abrupt jumps can be readily predicted. In particular we expect single mode jumps for quasistatic variations of the drive frequency in the sense leading to a crossing of the Eckhaus boundary, and the possibility of larger mode jumps for quasistatic sweeps in the reverse direction. This is qualitatively consistent with the numerical results of Lifshitz and Cross [19] for a numerical model of 67 parametrically driven resonators, who found more jumps in the solution on decreasing the frequency than on increasing it. (In the experiments of Buks and Roukes only upward frequency sweeps were performed.) A more detailed comparison with experiment would require a better knowledge of the parameters of the MEMS or NEMS devices so that the frequency variation could be mapped onto the reduced stability diagram of the amplitude equation. In future experiments, upward and downward sweeps of the strength of the driving would provide a more direct comparison with the theory we have developed.

\section{ACKNOWLEDGMENTS}

This work was funded by the U.S.-Israel Binational Science Foundation (BSF) through Grant No.2004339, the U.S. National Science Foundation under Grant No. DMR0314069, and the Israeli Ministry of Science and Technology.

\section{APPENDIX: EXPLICIT FORM OF SINGLE-MODE SOLUTIONS}

When substituting the single mode solution Eq. (7) with $\varphi=\pi / 4$ back into Eqs. (5) and (4), and then into Eq. (2), one obtains extended single-mode standing-wave parametric oscillations at half the drive frequency, whose explicit form is given by

$$
\begin{aligned}
u(x, t) \simeq & \epsilon^{1 / 2} \delta^{1 / 4} 4 S_{b} b_{k} \sin \left(q_{m} x\right) \\
& \times\left[\cos \left(\pi / 4-\omega_{p} t\right)+\tan (\alpha) \sin \left(\pi / 4-\omega_{p} t\right)\right] \\
= & \epsilon^{1 / 2} \delta^{1 / 4} 4 S_{b} b_{k} \sin \left(q_{m} x\right) \\
& \times\left[1+\tan ^{2}(\alpha)\right]^{1 / 2} \cos \left(\pi / 4-\omega_{p} t-\alpha\right),
\end{aligned}
$$

where we have defined

$$
\tan (\alpha) \equiv \delta^{1 / 2} \frac{4}{3} \omega_{p} \sin ^{4}\left(\frac{q_{p}}{2}\right)\left(b_{k}^{2}-k\right),
$$

and the scale factor 


$$
S_{b} \equiv \frac{4}{3 \sqrt{3}} \omega_{p} \sin ^{3}\left(\frac{q_{p}}{2}\right) .
$$

To satisfy the boundary conditions $u(0, t)=u(N+1, t)=0$, the wave numbers $q_{m}$ satisfy the equation

$$
q_{m}=\frac{m \pi}{N+1}=q_{p}+\frac{k \pi}{\Delta Q_{N}(N+1)},
$$

where

$$
\Delta Q_{N}=\frac{1}{\epsilon \delta^{1 / 2}} \frac{3 \Delta^{2} \sin \left(q_{p}\right)}{16 \omega_{p}^{2} \sin ^{6}\left(q_{p} / 2\right)} \frac{\pi}{N+1} .
$$

[1] R. Lifshitz and M. C. Cross, in Review of Nonlinear Dynamics and Complexity, edited by H. G. Schuster (Wiley, New York, 2008), p. 1.

[2] I. Bargatin, E. Myers, P. Andreucci, S. Hentz, J. Aldridge, and M. Roukes (private communication).

[3] K. L. Turner, S. A. Miller, P. G. Hartwell, N. C. MacDonald, S. H. Strogatz, and S. G. Adams, Nature (London) 396, 149 (1998).

[4] H. G. Craighead, Science 290, 1532 (2000).

[5] E. Buks and M. L. Roukes, Europhys. Lett. 54, 220 (2001).

[6] D. V. Scheible, A. Erbe, R. H. Blick, and G. Corso, Appl. Phys. Lett. 81, 1884 (2002).

[7] W. Zhang, R. Baskaran, and K. L. Turner, Sens. Actuators, A 102, 139 (2002).

[8] W. Zhang, R. Baskaran, and K. Turner, Appl. Phys. Lett. 82, 130 (2003).

[9] M.-F. Yu, G. J. Wagner, R. S. Ruoff, and M. J. Dyer, Phys. Rev. B 66, 073406 (2002).

[10] J. S. Aldridge and A. N. Cleland, Phys. Rev. Lett. 94, 156403 (2005).

[11] A. Erbe, H. Krommer, A. Kraus, R. H. Blick, G. Corso, and K. Richter, Appl. Phys. Lett. 77, 3102 (2000).

[12] I. Kozinsky, H. W. C. Postma, O. Kogan, A. Husain, and M. L. Roukes, Phys. Rev. Lett. 99, 207201 (2007).

[13] B. DeMartini, J. Rhoads, K. Turner, S. Shaw, and J. Moehlis, J. Microelectromech. Syst. 16, 310 (2007).

[14] S. C. Masmanidis, R. B. Karabalin, I. De Vlaminck, G. Borghs, M. R. Freeman, and M. L. Roukes, Science 317, 780 (2007).

[15] M. C. Cross, A. Zumdieck, R. Lifshitz, and J. L. Rogers, Phys. Rev. Lett. 93, 224101 (2004).

[16] M. C. Cross, J. L. Rogers, R. Lifshitz, and A. Zumdieck, Phys. Rev. E 73, 036205 (2006).
[17] E. Buks and M. L. Roukes, J. Microelectromech. Syst. 11, 802 (2002).

[18] M. Sato, B. E. Hubbard, and A. J. Sievers, Rev. Mod. Phys. 78, 137 (2006).

[19] R. Lifshitz and M. C. Cross, Phys. Rev. B 67, 134302 (2003).

[20] Y. Bromberg, M. C. Cross, and R. Lifshitz, Phys. Rev. E 73, 016214 (2006).

[21] X. M. H. Huang, C. A. Zorman, M. Mehregany, and M. L. Roukes, Nature (London) 421, 496 (2003).

[22] A. N. Cleland and M. R. Geller, Phys. Rev. Lett. 93, 070501 (2004).

[23] M. C. Cross and P. C. Hohenberg, Rev. Mod. Phys. 65, 851 (1993).

[24] L. Kramer, H. R. Schober, and W. Zimmermann, Physica D 31, 212 (1988).

[25] L. D. Landau and E. M. Lifshitz, Mechanics, 3rd ed. (Butterworth-Heinemann, Oxford, 1976), Sec. 27.

[26] G. Ahlers, M. C. Cross, P. C. Hohenberg, and S. Safran, J. Fluid Mech. 110, 297 (1981).

[27] M. B. Tarlie and K. R. Elder, Phys. Rev. Lett. 81, 18 (1998).

[28] Note that for the positive square-root branch (10) $b_{k}^{2}(k-1$ $\left.-b_{k}^{2}\right)<0$, implying that $\lambda_{g, k}(0)=0$, while for the negative square-root solution $b_{k}^{2}\left(k-1-b_{k}^{2}\right)>0$, implying that $\lambda_{g, k}(0)$ $>0$. As a consequence the positive square-root solution (10) is stable with respect to small perturbations with the same wave number $k$, while the negative square-root solution is unstable.

[29] The behavior when the saddle node instability is encountered on decreasing $g$ is expected to be similar for slow or rapid variation of $g$, since the dynamics is simply the decay of the mode to zero, with the subsequent growth of other modes.

[30] For slow ramps the instantaneous rate of growth of perturbations is well approximated by ignoring the time dependence of the parameter. 